\title{
Status and perspectives of fragmentation beams at LNS with CHIMERA detector
}

\author{
G. Cardella ${ }^{1}$, L. Acosta ${ }^{2,9}$, F. Amorini ${ }^{2}$, L. Auditore $^{4}$, \\ I. Berceanu ${ }^{7}$, M.B. ChatterjieE ${ }^{8}$, E. De Filippo ${ }^{1}$, \\ L. Francalanza ${ }^{2,3}$, L. Grassi ${ }^{1}$, E. La Guidara ${ }^{1}$, G. Lanzalone ${ }^{2,5}$, \\ I. Lombardo ${ }^{6}$, T. Minnitit $^{4}$, A. Pagano ${ }^{1}$, E.V. Pagano ${ }^{2,3}$, M. Papa $^{1}$, \\ S. Pirrone ${ }^{1}$, G. Politi ${ }^{1,3}$, L. Quattrocchi ${ }^{4}$, F. Rizzo ${ }^{2,3}$,

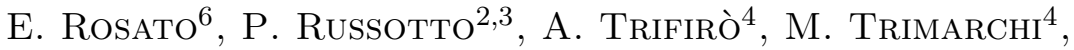 \\ G. VERDE $^{1}$ and M. Vigilante ${ }^{6}$ \\ ${ }^{1}$ INFN, Sezione di Catania, Catania, Italy \\ 2 INFN-LNS, Catania, Italy \\ 3 Dipartimento di Fisica e Astronomia Università di Catania, Italy \\ ${ }^{4}$ INFN, Gruppo Collegato di Messina and Dip. di Fisica Università di \\ Messina, Italy \\ ${ }^{5}$ Facoltà di Ingegneria and Architettura, Università Kore, Enna, Italy \\ 6 INFN, Sezione di Napoli and Dip. di Fisica Università di Napoli \\ Federico II, Italy \\ 7 National Institute for Physics and Nuclear Engineering \\ Bucharest-Măgurele, Romania \\ 8 Saha Institute for Nuclear Physics, Kolkata, India \\ ${ }^{9}$ Departamento de Fisica Aplicada, Universidad de Huelva, Huelva, Spain
}

\begin{abstract}
Relatively large yields of various exotic beams produced through in-flight fragmentation are available at LNS. Using the CHIMERA detector, we performed various experiments to study elastic and inelastic scattering, transfer, break-up, and reaction dynamics with targets from proton and deuteron to carbon and heavier. For reactions with relatively light systems we used the kinematical coincidence method to extract high resolution angular distributions of binary reactions from the measured light particle energy spectra. We also used the CsI detectors of CHIMERA to detect gamma rays emitted in the reactions. Some of most recent results are presented together with future perspectives with the coupling of CHIMERA with FARCOS array.
\end{abstract}




\section{Introduction}

After the recent intensity upgrade [1] of the LNS fragmentation beam, relatively large yields of various neutron rich and poor exotic beams, from 20 to $50 \mathrm{MeV} / \mathrm{A}$, are available. In order to better profit of such beams with CHIMERA detector [2,3] a tagging system [4] was developed to event by event identify the characteristics of the beam particles. Such system is going to be further implemented with a ppac position sensitive detector, in order to measure also the beam trajectory event by event. Using these beams, an ambitious experimental program was started, based on the study of both structure and dynamical effects on nuclear reactions mechanisms. Measurements on elastic scattering and neutron transfer reactions, on proton and deuteron targets, have been performed. Beams as ${ }^{10,11} \mathrm{Be},{ }^{13} \mathrm{~B},{ }^{16} \mathrm{C}$, produced by fragmentation of ${ }^{13} \mathrm{C}$ and ${ }^{18} \mathrm{O}$ at $55 \mathrm{MeV} / \mathrm{A}$ were used. The kinematical coincidence method was developed in order to extract angular distributions, with resolution of the order of $1^{\circ}$, in the center of mass frame (CM) [5]. New experiments are in program in the next future. The first one programmed (CLIR experiment) will use again the ${ }^{16} \mathrm{C}$ cocktail beam, produced by ${ }^{18} \mathrm{O}$ fragmentation, in order to study break-up reactions on ${ }^{12} \mathrm{C}$ target. With such reactions we want to study exotic structures as $\alpha-n$ chaines in nuclei as ${ }^{10} \mathrm{Be}$ or ${ }^{16} \mathrm{C}$ trying to improve recent results [6]. Another experiment will use the ${ }^{68} \mathrm{Ni}$ beam, produced by ${ }^{70} \mathrm{Zn}$ fragmentation, to study isoscalar excitation of the PIGMY resonance. For such experiments will be also used the prototype of the FARCOS detection system [7]. These detectors will be placed at forward angles to fully identify the projectile like fragments. Also $\gamma$-rays will be detected using the $\mathrm{CsI}(\mathrm{Tl})$ detectors of the CHIMERA sphere.

\section{Beam production, and tagging system}

The beam is produced mainly by fragmentation reactions, using the beams accelerated by the superconducting Cyclotron (CS) of the Laboratori Nazionali del Sud in Catania. A Beryllium target is placed, near the CS output channel, between two triplets of quadrupole. The quadrupole setting is optimized in order to produce the smallest possible beam spot on the target and to collect the maximum yield for the reaction fragments. The standard primary beam intensity, up to now used, is of the order of 100 Watts. The beam selection is obtained with appropriate setting of the first two $45^{\circ}$ dipoles of the main beam transport line of the CS. 


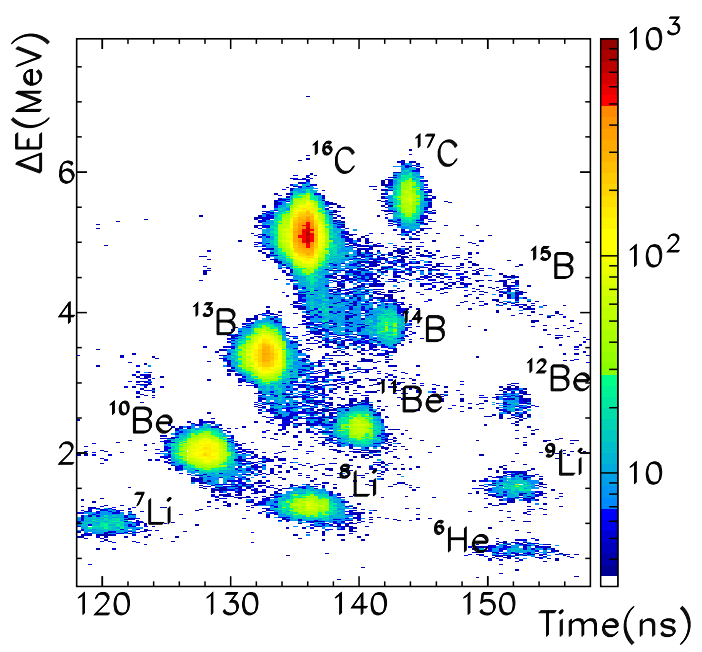

Figure 1: Identification scatter plot for the production of ${ }^{16} \mathrm{C} /{ }^{10,11}$ Be beams.

The tagging system is an evolution of the first tagging system used for fragmentation experiment at LNS [8]. It is based on the use of a 32x32 strips double side silicon strip detector (DSSSD) measuring the energy loss of the impinging beam particles. The identification of the particles is achieved combining this energy loss with the time of flight information (TOF) of the particle. The start of this TOF measurement is provided by a large size micro-channel plate (MCP) [4] mounted $13 \mathrm{~m}$ away from the DSSSD. The stop is given by the front strips of the DSSSD.

An example of the obtained identification scatter plot is shown in fig. 1 for the production of ${ }^{16} \mathrm{C}$ by ${ }^{18} \mathrm{O} 55 \mathrm{AMeV}$ beam fragmentation. To measure trajectories we have installed, at the entrance of the CHIMERA sphere, a PPAC position sensitive detector mounted on a remotely movable arm. The position information provided combining DSSSD and PPAC allows to extract the beam trajectory, and it is also very useful to sketch the beam profile at the target position.

\section{Kinematical coincidences method}

Using the $4 \pi$ CHIMERA detector it is relatively easy to detect all reaction partners, especially at low multiplicity, when multiple-hit of particles is negligible. In binary reaction on light targets as ${ }^{10} \mathrm{Be}+\mathrm{d}$ we will generally 


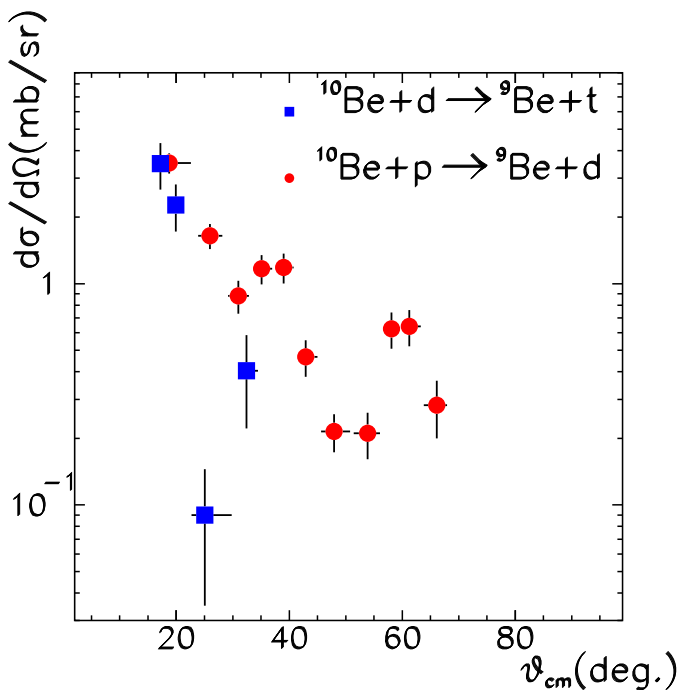

Figure 2: Center of mass angular distributions obtained for the reactions $\mathrm{p}\left({ }^{10} \mathrm{Be},{ }^{9} \mathrm{Be}\right) \mathrm{d}$ (circles) and $\mathrm{d}\left({ }^{10} \mathrm{Be},{ }^{9} \mathrm{Be}\right) \mathrm{t}$ (squares).

detect the heavy fragment at forward angles and the light partner at all angles allowed by kinematics. If one want to extract angular distributions in the CM for elastic or transfer reactions it is more convenient to look at the angular distribution of the light partner, because of its larger kinematic spread. Unfortunately CHIMERA measures this particle with a relatively bad angular resolution $\left( \pm 4^{\circ}\right.$ in the worse case at angles larger than $30^{\circ}$ in the CHIMERA spherical region). This produces an even worse angular resolution in CM. However we know that there is a strict correlation, approximately linear in a large angular range, between the CM emission angle and the energy of the light particle. Therefore because we can measure with relatively good resolution (better than $2 \%$ ) the light particle energy, using kinematic rules it is quite simple to transform energy spectra in CM angular distributions with the same percentage error. Obviously one has to evaluate, with simulations, solid angle, efficiency and the Jacobian of the transformation. We emphasize that this method automatically correct for the spread of the beam trajectory. Examples of the angular distribution we can extract, using this method, are given in fig.2 (adapted from ref. [5]). In such figure we plot the angular distributions obtained in the $\mathrm{p}\left({ }^{10} \mathrm{Be},{ }^{9} \mathrm{Be}\right) \mathrm{d}$ (circles) and $\mathrm{d}\left({ }^{10} \mathrm{Be},{ }^{9} \mathrm{Be}\right) \mathrm{t}$ (squares) reactions. Coupled channel calculations are in progress in order to compare such results with previously measured 


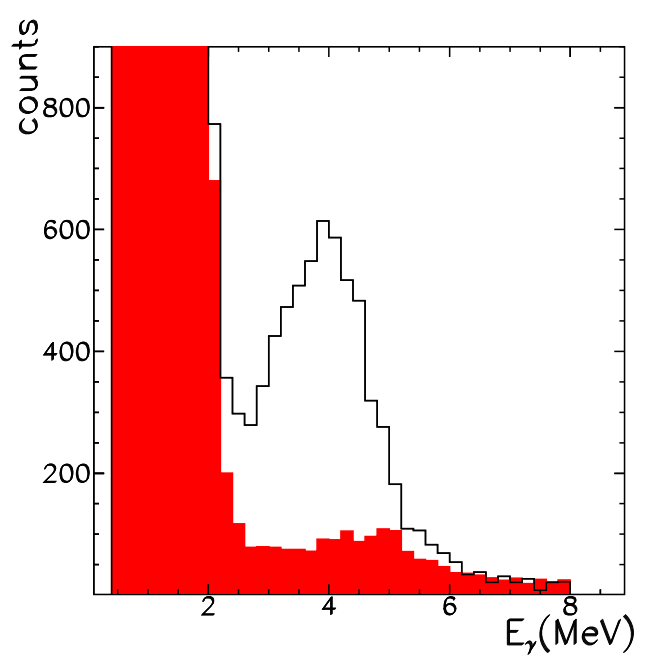

Figure 3: $\gamma$-ray spectrum detected in CHIMERA $\mathrm{CsI}(\mathrm{Tl})$ in coincidence with proton from elastic scattering on carbon target (filled spectrum), and in coincidence with proton from inelastic scattering leaving the carbon excited at the $4.44 \mathrm{MeV}$ level (empty spectrum).

direct $\mathrm{d}$, t reactions on ${ }^{10} \mathrm{Be}$ targets at $15 \mathrm{MeV}$ and to compare the potential extracted with transfer reactions to the ones tat can be obtained in direct knock-out reactions $[9,10]$.

\section{Gamma ray detection}

In reactions with radioactive beams, due to the low beam intensity, it is today mandatory to perform measurement as complete as possible. It is for instance very important to be able to detect the $\gamma$-rays in coincidence with particles emitted during the reaction. With CHIMERA we detect and identify $\gamma$-rays in all $\mathrm{CsI}(\mathrm{Tl})$ detectors. However we have a better signal to noise ratio in the detectors of the spherical part. Such detectors, in fact, cover a larger solid angle and have a smaller particle yield, due to kinematical focusing. We measured the $\mathrm{CsI}(\mathrm{Tl})$ response function to $\gamma$ rays, looking for instance to the detection of the well know $4.44 \mathrm{MeV} \gamma$-ray, emitted during the decay stage of the first excited state of ${ }^{12} \mathrm{C}$. This state can be simply excited using proton scattering. In such kind of reactions one can look for the detection of this $\gamma$-ray triggering the event with the 
inelastic scattered proton. This is a way to measure also the efficiency. In fig. 3 we show the spectrum collected in one ring of the sphere, in coincidence with such inelastic scattering events (empty spectrum). Background can be evaluated collecting the same number of events, triggered by the detection of protons from elastic scattering (full spectrum). One can see that the peak to noise ratio is rather good. Resolution is of the order of $0.5 \mathrm{MeV}$, as can be deduced by the missing observation of the first and second escape peaks. From comparison with GEANT-IV simulations, one understand that the energy response function is centered on the first escape peak at about 3.9 $\mathrm{MeV}$. We have also verified that we can simply extract angular distributions from such events. They are very useful to understand the multipolarity of the emitted $\gamma$-ray, so obtaining information about the spin of the observed excited level. The analysis on the $\gamma$-ray collected with fragmentation beams is going. We hope to get soon new spectroscopic information about the populated systems.

\section{$5 \quad$ Future perspectives}

During next measurements, we will improve the angular and energy resolution in the detection of the heavy projectile like fragments, by using the highly segmented, first four prototypes, of the FARCOS detection system [7]. A FARCOS module is a three stage telescope composed by two DSSSD, 32 strips for each side, 300 and $1500 \mu \mathrm{m}$ thick, with a total surface of $64 \times 64$ $\mathrm{mm}^{2}$. These DSSSD are followed by four $\mathrm{CsI}(\mathrm{Tl})$ detectors with a surface of $32 \times 32 \mathrm{~mm}^{2}$ each one, $6 \mathrm{~cm}$ thick, with photo-diode readout, for the detection of light particles. The two stages of silicon detectors guarantee a much better energy resolution than CHIMERA telescopes. Also the angular resolution will be much better, due to the $2 \times 2 \mathrm{~mm}^{2}$ size of the detection pixels. Also the $\mathrm{CsI}(\mathrm{Tl})$, due to the smaller size, and the better doping uniformity, have an energy resolution improved respect to CHIMERA CsI(Tl). Moreover, due to the DSSSD pixellation, we can correct for position dependence of $\mathrm{CsI}(\mathrm{Tl})$ light response, so further improving energy resolution. This will allow to study, with very good accuracy, excited levels of ${ }^{10} \mathrm{Be}$ or ${ }^{16} \mathrm{C}$ and other nuclei of the cocktail beam reconstructed after the break up on carbon targets (CLEAR experiment). Preliminary analysis performed on previously collected data [11], shows the possibility to extract new information on a rotational band of ${ }^{10} \mathrm{Be}$ and also on never seen high energy levels of ${ }^{16} \mathrm{C}$. The use of FARCOS will be also mandatory for the event selection on next PIGMY experiment. We will study the possible excitation, 
via isoscalar probe [12], of the pigmy resonance in the ${ }^{68} \mathrm{Ni}$. In this experiment we will detect $\gamma$-rays in the CHIMERA $\mathrm{CsI}(\mathrm{Tl})$ in coincidence with ${ }^{68} \mathrm{Ni}$ inelastic scattering. We will also try to detect neutrons emitted from the decay of the same resonance. Such neutrons will be measured in the CsI detectors of forward CHIMERA telescopes. Charged particles cannot be in fact detected in such telescopes, because they will be screened by FARCOS telescopes. We have recently verified that, in this way, we can clearly observe the charged particles produced by neutron interaction with CsI material, so detecting the neutrons. We can conclude that using CHIMERA and FARCOS telescopes we have a very beautiful complete instrument to detect at the same time light particles, heavy fragments, neutrons and $\gamma$-rays, opening very large opportunities for future experiments. We hope in the next future also to have a further upgrading of the intensity of the fragmentation beam with the proposed replacement and rebuilding of the CS cryostat, allowing for a primary beam intensity upgrade up to 2 orders of magnitude.

\section{References}

[1] see http://fribs.lns.infn.it/upgrade-results.html

[2] A.Pagano et al Nucl.Phys. A 734 (2004) 504 and ref. therein

[3] A.Pagano, Nuclear Physics News International, 22:1(2012)25.

[4] I. Lombardo et al Nucl.Phys.B (Proc.Suppl.) 215(2011)272

[5] L.Acosta et al NIM A 715 (2013) 56

[6] M. Freer et al., Phys. Rev. Lett. 96, 042501 (2006).

[7] G.Verde et al Journ. of Phys.: Conf. Ser. 420 (2013) 012158

[8] G.Raciti et al Phys.Rev.Lett. 100, 19250(2008).

[9] N. Keeley, K.W. Kemper, K. Rusek, Physical Review C 86 (2012) 014619 .

[10] G. F. Grinyer et al Phys. Rev. Lett. 106, 162502 (2011).

[11] D.Dell'Aquila degree thesis Catania University.

[12] E. G. Lanza, A. Vitturi, M. V. Andres, F. Catara and D. Gambacurta, Phys. Rev. C 84, 064602 (2011). 